\title{
The Effect of Dexpanthenol on Ototoxicity Induced by Cisplatin
}

\author{
Yuksel Toplu ${ }^{1}$ Emrah Sapmaz ${ }^{2}$ Hakan Parlakpinar ${ }^{3} \cdot$ Mehmet Kelles $^{4} \cdot$ M. Tayyar Kalcioglu ${ }^{5} \cdot$ Kevser Tanbek $^{6} \cdot$ Ahmet Kizilay $^{1}$ \\ ${ }^{I}$ Department of Otorhinolaryngology, Inonu University Medical Faculty, Malatya; ${ }^{2}$ Department of Otorhinolaryngology, Malatya State Hospital, \\ Malatya; ${ }^{3}$ Department of Pharmacology, Inonu University Medical Faculty, Malatya; ${ }^{4}$ Department of Otorhinolaryngology, Sutcu Imam \\ University Medical Faculty, Kahramanmaras; ${ }^{5}$ Department of Otorhinolaryngology, Istanbul Medeniyet University Medical Faculty, Istanbul; \\ ${ }^{6}$ Department of Physiology, Inonu University Medical Faculty, Malatya, Turkey
}

Objectives. This study was aimed to investigate the protective effects of dexpanthenol (Dxp) on against cisplatin-induced ototoxicity.

Methods. To examine this effect, distortion product otoacoustic emissions (DPOAEs) measurements and serum levels of oxidative and antioxidant status (including malondialdehyde, superoxide dismutase, catalase, glutathione, glutathione peroxidase, total oxidant status, total antioxidant status, and oxidative stress index) were evaluated. Thirty-two adult female Wistar albino rats were randomly divided into 4 equal groups; control (K), cisplatin (C), cisplatin plus Dxp (CD), and Dxp (D). In all groups DPOAEs measurements, between 996 and 10,078 Hz as DPOAEs and input/output functions, were performed on days 0, 1th, 5th, and 12th. Prior to death, the last DPOAEs measurements and blood samples were taken.

Results. In the $\mathrm{C}$ group, statistically significant differences were detected at all frequencies between 0 and 5 days and 0 and 12 days measurements $(P<0.05)$. Serum level of oxidant and antioxidant status were detected statistically significantly changed in this group versus $\mathrm{K}$ group $(P<0.05)$. Contrary to the $\mathrm{C}$ group, in the $\mathrm{CD}$ group hearing ability was seen largely preserved at many frequencies and serum levels of all biochemical parameters were shifted toward normal values, similar to the K group. No significant differences were detected in the either D or K group's measurements.

Conclusion. According to these results, Dxp may prevent cisplatin-induced ototoxicity.

Keywords. Cisplatin; Ototoxicity; Dexpanthenol; Otoacoustic Emissions; Biomarkers

\section{INTRODUCTION}

Cisplatin is a commonly used antineoplastic agent that treats many neoplastic diseases, including ovarian, testicular, lung, and head and neck cancer. The antineoplastic mechanism is related

- Received Jnne 24, 2014

Revised January 21, 2015

Accepted February 26, 2015

- Corresponding author: Emrah Sapmaz

Department of Otorhinolaryngology, Malatya State Hospital, Özalper Mah.

Turgut Özal Bulvarı No:4 44330, Malatya,Turkey

Tel: +90-422-212-3611, Fax: +90-422-341-0128

E-mail: emrhils@yahoo.com to inhibition of DNA synthesis [1]. In addition to its efficiency against cancer, cisplatin also has severe side effects that limit its clinical use, including ototoxicity, nephrotoxicity, and neurotoxicity [2].

Cisplatin-induced ototoxicity (CIO) was first defined by Rossof et al. [3] in 1972. Ototoxicity may occur within hours to days following cisplatin administration. CIO causes sensorineural hearing loss (primarily at high frequencies) that is frequently bilateral, progressive, and irreversible. Hearing loss may progress and involve all frequencies secondary to the dose and frequency of drug administration [4].

The mechanism of CIO is still not exactly understood [5]. The

Copyright (C) 2016 by Korean Society of Otorhinolaryngology-Head and Neck Surgery.

This is an open-access article distributed under the terms of the Creative Commons Attribution Non-Commercial License (http://creativecommons.org/licenses/by-nc/4.0)

which permits unrestricted non-commercial use, distribution, and reproduction in any medium, provided the original work is properly cited. 
generation of reactive oxygen species (ROS), leading to cellular damage in the cochlea, probably plays a significant role [6]. Cisplatin administration also decreases the presence of antioxidant enzymes in the cochlea [7]. Oxidative stress can occur when the balance between the production of ROS and antioxidative defense is damaged, possibly leading to cochlear cell injury or death. The use of antioxidant agents may prevent the hazardous effects of ROS [8] and has been reported in the literature [7]; therefore, use of antioxidant agents may be one logical approach to preventing CIO.

Dexpanthenol (Dxp, or D-panthenol; (+)-2,4-dihydroxy-N-(3hydroxypropyl)-3,3 dimethylbutyramide) is an alcoholic analog of pantothenic acid (PA) and is oxidized to PA in the peripheral tissues [9]. It is well recognized that PA and its derivatives can increase the levels of reduced glutathione (GSH), coenzyme A (Co A, especially mitochondrial Co A), and adenosine-5'-triphosphate (ATP) synthesis within the cell [10]. All of these play a major role in cellular protection and in the healing systems that work against oxidative stress and inflammatory response [11].

In this experimental rat model study, we investigated the potential protective and/or therapeutic effects of Dxp on the CIO by evaluating distortion product otoacoustic emissions (DPOAEs) measurements and serum levels of oxidative stress parameters (malondialdehyde [MDA], total oxidant status [TOS] and oxidative stress index [OSI]), and antioxidant contents (superoxide dismutase [SOD], catalase [CAT], glutathione peroxidase [GPX], reduced GSH, and total antioxidant status [TAC]). To our knowledge, the protective effect of Dxp on CIO has not yet been investigated in the literature.

\section{MATERIALS AND METHODS}

The study was performed according to the approved Experimental Animal Ethics Committee (2013/A-83) and was conducted in accordance with the Animal Welfare Act and the Guide for the Care and Use of Laboratory Animal (NIH publication No. 53773, 1996, Animal Ethics Committee). A total of 32 female Wistar albino rats, 3 months of age and with weight between 200 and $280 \mathrm{~g}$, were used. Experimental animals were housed under automatically regulated conditions to have 12 hours in the light and 12 hours in the dark per day at an ambient temperature of $21^{\circ} \mathrm{C} \pm 1^{\circ} \mathrm{C}$ with $45 \%$ to $50 \%$ humidity. All animals were fed with standard pellets and were given fresh tap water daily.

Rats were anesthetized with $50-\mathrm{mg} / \mathrm{kg}$ intramuscular ketamine and $5-\mathrm{mg} / \mathrm{kg}$ intramuscular xylazine. Each rat underwent a careful examination of its external ear and tympanic membranes. The model rats were then brought into special noiseless cabins (sound pressure level not exceeding $45 \mathrm{~dB}$ ) for the presentation of subsequent procedures. Before starting the study, each rat was screened with otoacoustic emission measurements to assess hearing function. For otoacoustic emission measurements, DPOAE values were used. The rats with normal DPOAE findings were included in the study.

Rats were divided into 4 groups by randomization; control $(\mathrm{K})$, cisplatin (C), cisplatin plus Dxp (CD), and Dxp (D). The K group included 8 rats, which received intraperitoneal (i.p.) saline at daily doses of $5 \mathrm{~mL}$ for 5 days. The $\mathrm{C}$ group comprised 8 rats that received 16-mg/kg (i.p.) cisplatin single dose (cisplatin DBL, $50 \mathrm{mg}$, Orna Corp., Istanbul, Turkey) at day 0. The CD group consisted of 8 rats and received cisplatin 16-mg/kg (i.p.) single dose at day 0 plus Dxp (500 mg/kg i.p. daily doses for 5 days). The D group contained 8 rats and received Dxp (500 mg/kg i.p. daily doses for 5 days) (Sigma Chemical Co., St Louis, MO, USA). DPOAE measurements were performed in all groups on days $0,1,5$, and 12 .

The dosages of cisplatin and Dxp were determined based on results from previous dose-response studies that reported ototoxicity (related to cisplatin) and marked antioxidative and antiinflammatory effects (related to Dxp) in rats [5,12].

DPOAE measurements were performed using a GSI Audera DPOAE device (Grason Stadler, Madison, WI, USA). The otoacoustic emission probe was placed and calibrated using an automated measurement system before each test, then positioned in the external ear canal. The measurements were performed in a quiet environment. Primary stimulus levels were equalized at $65 \mathrm{~dB}$ (L1=L2). Two different frequencies (f1 and f2) were prepared at $\mathrm{f} 2: \mathrm{f} 1=1.22$ for taking the most powerful responses. DPOAE measurements were performed at frequencies of 996, 1,266, 1,582, 2,004, 2,519, 3,176, 3,996, 5,039, 6,351, 8,004, and $10,078 \mathrm{~Hz}$ and the results were noted. All animals were killed under general anesthesia on day 12. Before death, the last otoacoustic emission measurements and blood samples (for serum levels of MDA, SOD, CAT, GPX, GSH, TOS, TAC, and OSI) were taken.

\section{Biochemical analyses}

The MDA contents of the homogenates were calculated spectrophotometrically by evaluating the presence of thiobarbituric acid-reactive substances [13]. The results were expressed in nmol/ $\mathrm{mL}$ according to a prepared standard graph.

Total SOD activity was measured according to the method of Sun et al. [14]. The principle of the method is the inhibition of nitroblue tetrazolium reduction by the xanthine-xanthine oxidase system as a superoxide generator. SOD activity was expressed as U/mg protein. CAT activity was measured using the method developed by Aebi [15]. The principle of the assay is based on the determination of the rate constant $(k, s-1)$ or the $\mathrm{H}_{2} \mathrm{O}_{2}$ decomposition rate at $240 \mathrm{~nm}$. Results were expressed as $\mathrm{k} / \mathrm{g}$ protein. GPX activity was calculated according to a method developed by Paglia and Valentine [16]. An enzymatic reaction in a tube containing nicotinemide adenine dinucleotide phosphate, GSH, sodium azide, and glutathione reductase was initiated by adding $\mathrm{H}_{2} \mathrm{O}_{2}$; the change in absorbance at $340 \mathrm{~nm}$ was 
observed using a spectrophotometer. Activity was expressed as $\mathrm{U} / \mathrm{mg}$ protein. The GSH content in the serum as nonprotein sulfhydryls was evaluated using a previously described method [17]. The absorbance values were extrapolated from a glutathione standard curve and expressed as GSH ( $\mu \mathrm{mol} / \mathrm{L})$. Serum TAC levels were measured using a novel automated measurement method developed by Erel [18]; this assay is excellent from a precision standpoint, with precision values lower than 3\%. The results are expressed as $\mu$ mol Trolox equivalent/L. Serum TOS levels were calculated using a novel automated measurement method developed by Erel [19]. The assay is calibrated $\mathrm{H}_{2} \mathrm{O}_{2}$ and the results are expressed as $\mu$ mol $\mathrm{H}_{2} \mathrm{O}_{2}$ Trolox equivalent/L. The TOS: TAC ratio was used to measure OSI, and the results are expressed in arbitrary units [20].

\section{Statistical analysis}

For detecting even minor effects, the required sample sizes used in this experiment were identified using statistical power analysis. The sample sizes necessary for a power of 0.80 were estimated using NCSS software (Kaysville, UT, USA). Data were analyzed using the IBM SPSS Statistics ver. 22.0 (IBM Co., Armonk, NY, USA). The normality of the distribution was confirmed using the Kolmogorov-Smirnov test. The Wilcoxon test was used to compare DPOAE measurements with anormal distribution in the study groups; the Kruskal-Wallis and Mann-Whitney U-tests were used to compare differences between groups. Within group comparisons of parameters were performed using the Wilcoxon sign test. Kruskal-Wallis $H$-test, a Conover test were used to compare differences among the biochemical parameters. $P$-values less than 0.05 were regarded as statistically significant. The values were given as median (range).

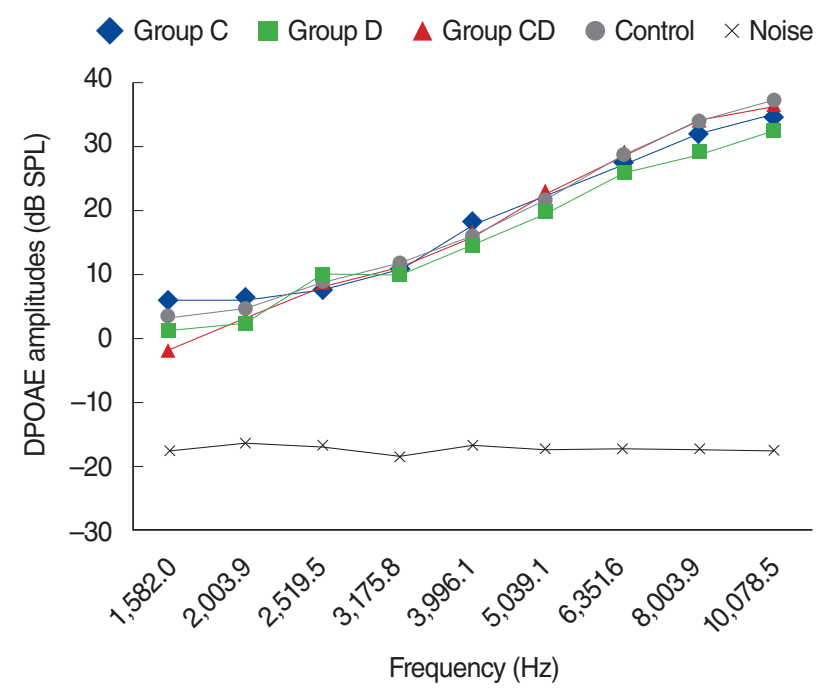

Fig. 1. Distortion product otoacoustic emission (DPOAE) amplitudes obtained from on day zero. C, cisplatin; D, dexpanthenol; CD, dexpanthenol plus cisplatin; SPL, sound pressure level.

\section{RESULTS}

The results from the DPOAE measurements for all groups (both before and after administration of Dxp and/or cisplatin) are presented in Figs. 1-4.

When group $\mathrm{C}$ were compared with group $\mathrm{K}$, there was a statistically significant differences between groups $(P<0.05)$. Group CD were compared with group $C$ at 1,5 , and 12 days there was a statistically significant differences between groups $(P<0.05)$ but group $\mathrm{CD}$ were compared with group $\mathrm{K}$ there was no statistically significant differences $(P>0.05)$.

In the $\mathrm{K}$ group, DPOAE measurements were performed at 0 , 1,5 , and 12 days; no statistical difference was shown for all frequencies $(P>0.05)$.

In the $C$ group, one of the 8 rats died because of enteritis and weight loss on the fifth day, possibly secondary to cisplatin toxicity. The other 7 rats completed the study and were evaluated for further analyses. Statistically significant differences were detected for all dosing frequencies between 0 and 5 days and 0 and 12 days $(P<0.05)$ (Fig. 5). Statistically significant differences were also detected at frequencies of 1,266, 1,582, 2,003, 3,175, 3,996, 5,039, 6,351, 8,003, and 10,078 Hz for measurements between 1 and 5 days, and at frequencies of 3,175, 3,996, 5,039, 6,351, 8,003, and $10,078 \mathrm{~Hz}$ between 5 and 12 days measurements $(P<0.05)$. However, no statistically significant difference surfaced for the measurements between 0 and 1 days $(P>0.05)$ (Fig. 5).

In the $\mathrm{CD}$ group, one of the 8 rats died because of anesthesia as measurements were being obtained during the second day. The other 7 rats were included in the evaluation process. No statistically significant differences were present between measurements obtained at 0 day and 1 day, or at 1 day and 5 days $(P>$

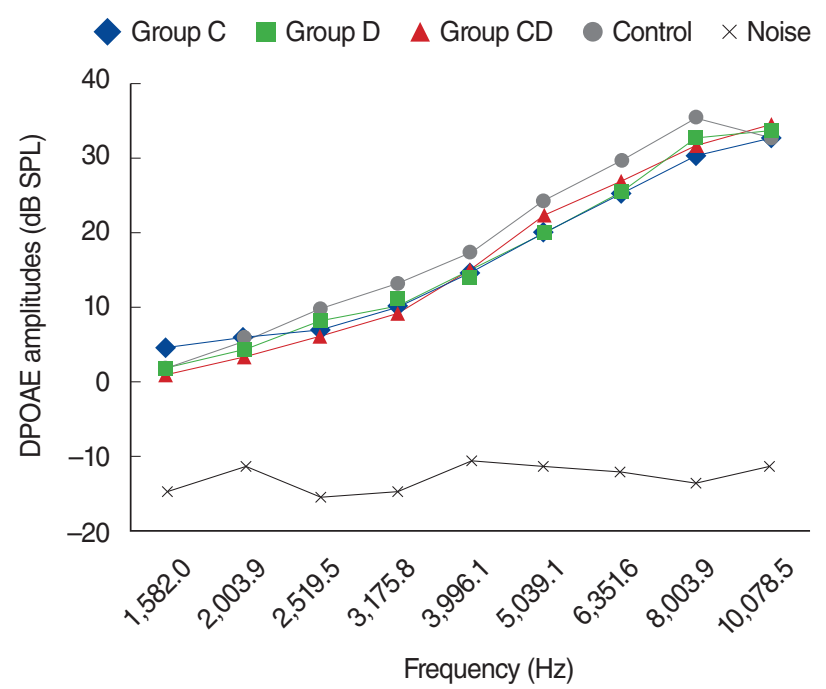

Fig. 2. Distortion product otoacoustic emission (DPOAE) amplitudes obtained on day 1. C, cisplatin; D, dexpanthenol; $C D$, dexpanthenol plus cisplatin; SPL, sound pressure level. 
0.05) (Fig. 6). Statistically significant differences were detected at frequencies 8,003 and 10,078 $\mathrm{Hz}$ for measurements obtained between days 0 and 5 and at frequencies 6,351, 8,003, and $10,078 \mathrm{~Hz}$ for measurements between days 0 and $12(P<0.05)$. Contrary to the $\mathrm{C}$ group, in the $\mathrm{CD}$ group hearing ability was largely preserved at many frequencies (Fig. 6).

The findings in the $\mathrm{D}$ group were similar to those shown in the K group. DPOAE measurements were performed on days 0 , 1,5 , and 12 , and there was no statistically significant difference

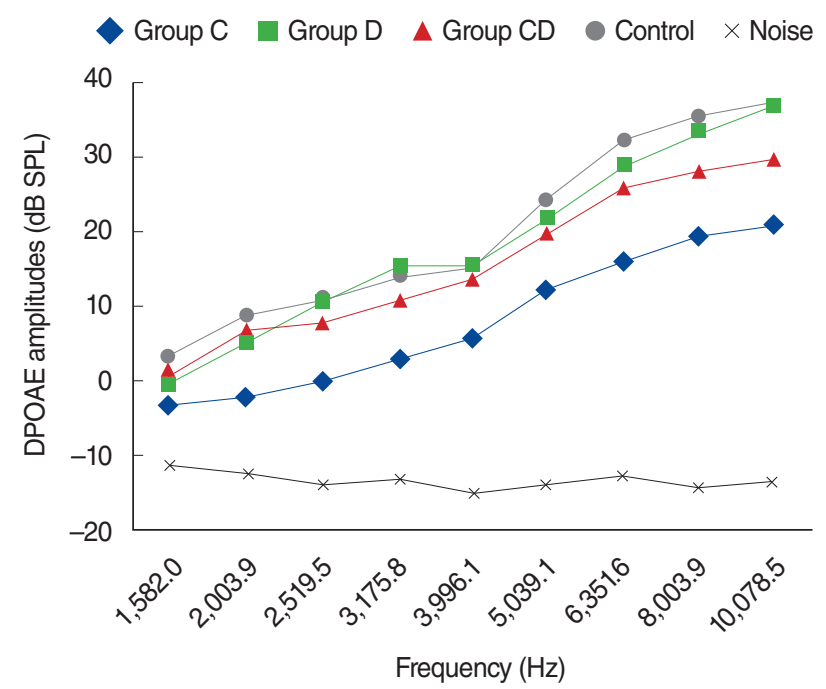

Fig. 3. Distortion product otoacoustic emission (DPOAE) amplitudes obtained on day 5. C, cisplatin; D, dexpanthenol; CD, dexpanthenol plus cisplatin; SPL, sound pressure level.

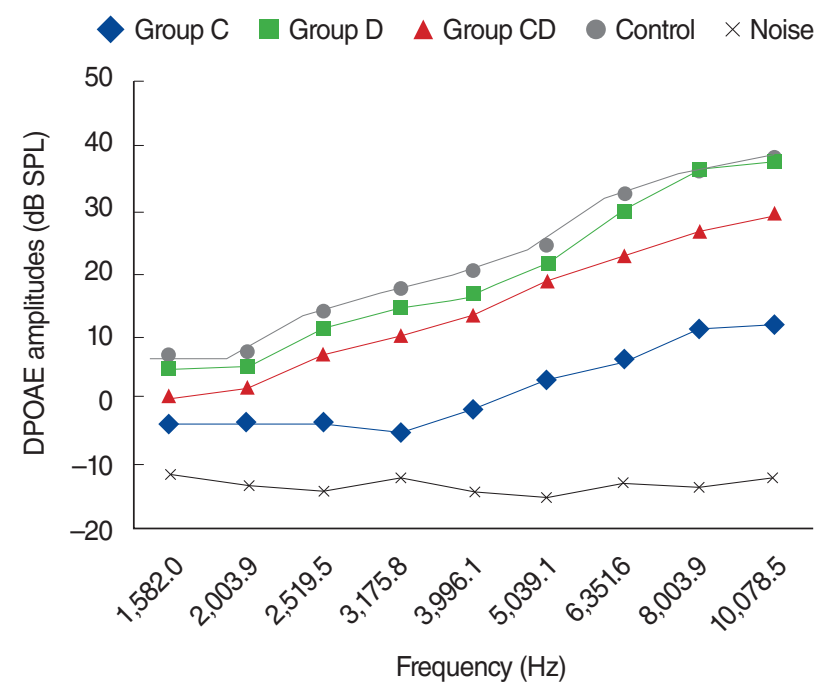

Fig. 4. Distortion product otoacoustic emission (DPOAE) amplitudes obtained on day 12. C, cisplatin; D, dexpanthenol; CD, dexpanthenol plus cisplatin; SPL, sound pressure level. at all frequencies $(P>0.05)$ (Fig. 7).

The serum levels of biochemical parameters are presented in Table 1. In brief, MDA, TOS, and OSI levels were significantly higher in the $\mathrm{C}$ group when compared to the $\mathrm{K}$ group, whereas Dxp supplementation with cisplatin (CD group) significantly reduced all of these toxicity parameters. Also, serum SOD, CAT, GPX, and TAC contents were significantly reduced in the C group, whereas Dxp treatment significantly improved the levels for all of these parameters.

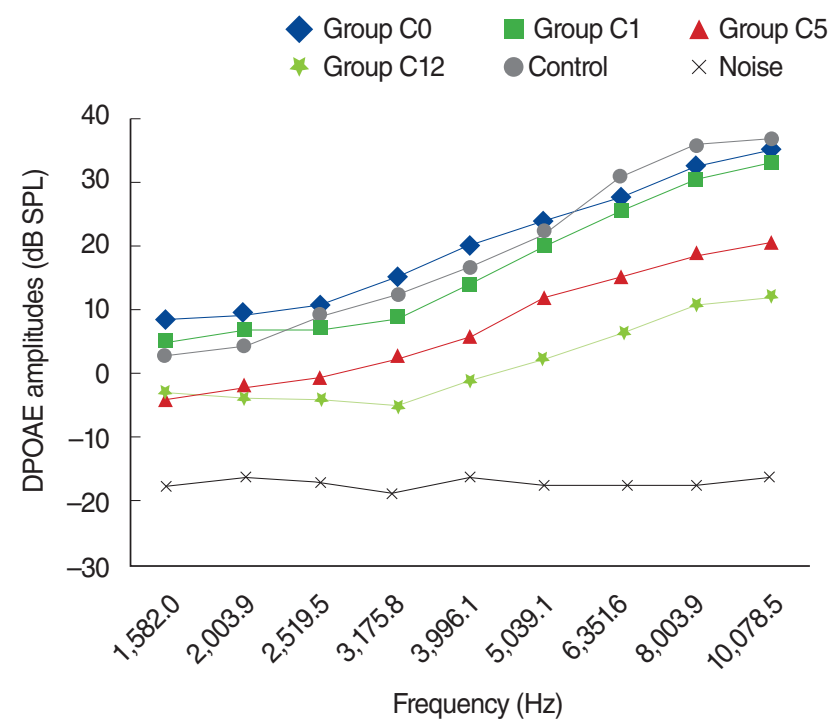

Fig. 5. Variations in amplitudes of distortion product otoacoustic emission (DPOAE) for different time points in cisplatin (C) group (C0, baseline [day 0]; C1, 1st day: C5, 5th day; C12, 12th day). SPL, sound pressure level.

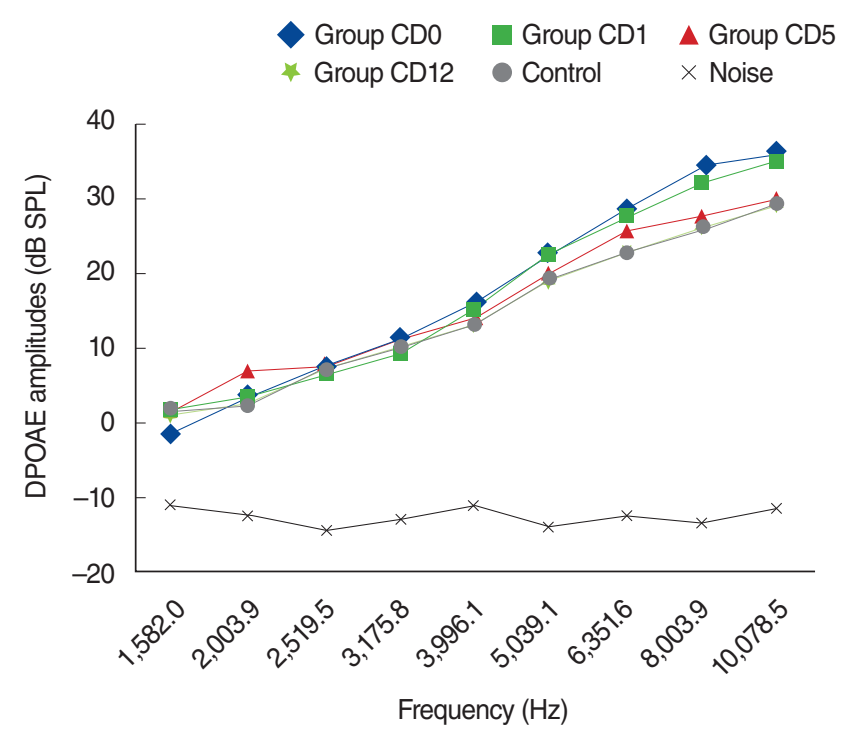

Fig. 6. Variations in amplitudes of distortion product otoacoustic emission (DPOAE) for different time points in cisplatin plus dexpanthenol (CD) used group (CD0, baseline [day 0]; CD1, 1st day; CD5, 5th day; CD12, 12th day). SPL, sound pressure level. 


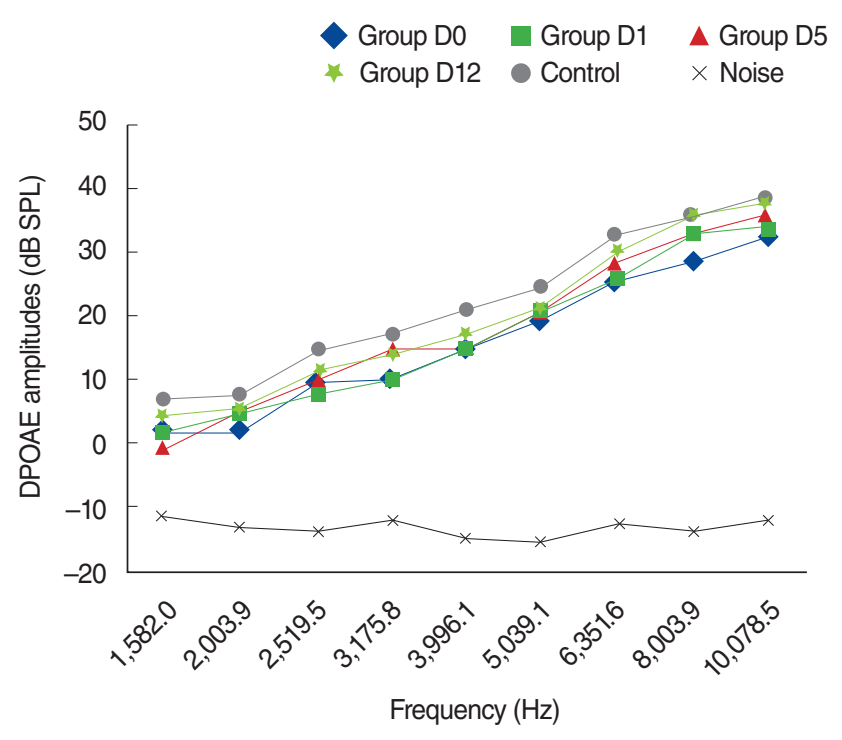

Fig. 7. Variations in amplitudes of distortion product otoacoustic emission (DPOAE) for different time points in dexpanthenol (D) group (D0, baseline [day 0]; D1, 1st day; D5; 5th day; D12, 12th day). SPL, sound pressure level.

\section{DISCUSSION}

Various opinions have been detailed in the literature concerning the mechanism of drug ototoxicity. According to Sagit et al. [21], the pathogenesis of drug ototoxicity may be due to the accumulation of drugs within cells. Another view is that the mechanism of cisplatin ototoxicity is based on ROS production, which affects antioxidant protection of the organ of Corti [22]. ROS reactions with cell membrane lipids produce toxic aldehydes. These aldehydes are implicated in apoptotic cell death machinery in the organ of Corti and the spiral ganglion, consequently causing inner ear hair cell degeneration [23].

In vitro studies have shown that cisplatin leads to hearing loss by affecting numerous regions of the cochlea. Outer hair cell destruction is the most commonly described manifestation of this ototoxicity. The stereocilia of outer hair cells are initially injured, followed by a loss of outer hair cells from the base to the apex. Damage also includes the collapse of Reissner's membrane, atrophy of the stria vascularis and supporting cells of the organ of Corti [24]. Although there are several reports demonstrating involvement of destructive free radical uptake in CIO, the mechanism of this ototoxicity remains to be completely explained [23].

In this study we used DPOAEs, which are highly selective for detecting cochlear hearing loss. The most important features of DPOAEs are their noninvasive capability and objectivity during the process of defining the early stages of sound processing and assessing the biomechanical activity of the outer hair cells [6]. DPOAE measurement is a well-defined method for detecting the effects of cisplatin on the cochlea before changes are identified by pure tone audiometry [25]. In the current study, statisti- 
cally significant reductions in DPOAEs amplitudes were noted at almost all frequencies in the $\mathrm{C}$ group; this seems to be strongly related to $\mathrm{CIO}$.

It is well established that antioxidants protect cells against the side effects of drugs and toxic radical reactions [26]. Currently, various antioxidants are focused on therapeutic purposes. Recombinant SOD, A-C-E vitamins, beta-carotene, glutathione, erdostein, aminoguanidine, and angiotensin converting enzyme inhibitors can be specified [27,28].

Dxp is oxidized to PA in the tissues. Previous studies have reported the protective effect of PA and its derivatives against cell destruction produced by oxygen free radicals [9,10,29]. PA and its derivatives increase the levels of reduced GSH and Co A as well as ATP synthesis within the cell [10,30]. Finally, all of these play a major role in cellular protection and in healing systems against oxidative stress and inflammatory response. Taking into consideration the reduced oxidative damage caused by Dxp treatment, all investigators attributed the protective actions of Dxp to its antioxidative and anti-inflammatory activities [11].

Our findings parallel those of earlier studies regarding Dxprelated antioxidant and anti-inflammatory effects. In the current study, the CIO was shown by measuring DPOAEs and biochemical parameters. The degree of ototoxicity induced by a single dose of cisplatin was significantly decreased in the CD group by Dxp treatment (Figs. 1-4). The current study differs from other ototoxicity studies on the same subject as we also assessed serum biochemical parameters, including new oxidant and antioxidant contents such as TOS, TAC, and OSI. Our biochemical results were in accordance with DPOAE measurements. We propose that Dxp acts in the body (including the ear) as a potent scavenger of free radicals and that its anti-inflammatory effects help prevent the toxic effects of cisplatin in the light of biochemical and DPOAE measurements. We believe Dxp could be effectively combined with cisplatin therapy in the selected cases.

In conclusion, the present study showed for the first time that Dxp, a safe and widely available agent, significantly prevented $\mathrm{CIO}$ in rats. However, further studies are required to evaluate the role of different doses of Dxp in the prevention and the treatment of CIO.

\section{CONFLICT OF INTEREST}

No potential conflict of interest relevant to this article was reported.

\section{REFERENCES}

1. Williams CJ, Whitehouse JM. Cis-platinum: a new anticancer agent. Br Med J. 1979 Jun;1(6179):1689-91.

2. Rabik CA, Dolan ME. Molecular mechanisms of resistance and tox- icity associated with platinating agents. Cancer Treat Rev. 2007 Feb; 33(1):9-23.

3. Rossof AH, Slayton RE, Perlia CP. Preliminary clinical experience with cis-diamminedichloroplatinum (II) (NSC 119875, CACP). Cancer. 1972 Dec;30(6):1451-6.

4. Kopelman J, Budnick AS, Sessions RB, Kramer MB, Wong GY. Ototoxicity of high-dose cisplatin by bolus administration in patients with advanced cancers and normal hearing. Laryngoscope. 1988 Aug; 98(8 Pt 1):858-64.

5. Kalcioglu MT, Kizilay A, Gulec M, Karatas E, Iraz M, Akyol O, et al. The protective effect of erdosteine against ototoxicity induced by cisplatin in rats. Eur Arch Otorhinolaryngol. 2005 Oct;262(10):856-63.

6. Lopez-Gonzalez MA, Guerrero JM, Rojas F, Delgado F. Ototoxicity caused by cisplatin is ameliorated by melatonin and other antioxidants. J Pineal Res. 2000 Mar;28(2):73-80.

7. Campbell KC, Rybak LP, Meech RP, Hughes L. D-methionine provides excellent protection from cisplatin ototoxicity in the rat. Hear Res. 1996 Dec;102(1-2):90-8.

8. Campbell KC, Meech RP, Rybak LP, Hughes LF. The effect of D-methionine on cochlear oxidative state with and without cisplatin administration: mechanisms of otoprotection. J Am Acad Audiol. 2003 Apr;14(3):144-56.

9. Ebner F, Heller A, Rippke F,Tausch I.Topical use of dexpanthenol in skin disorders. Am J Clin Dermatol. 2002;3(6):427-33.

10. Slyshenkov VS, Piwocka K, Sikora E, Wojtczak L. Pantothenic acid protects jurkat cells against ultraviolet light-induced apoptosis. Free Radic Biol Med. 2001 Jun;30(11):1303-10.

11. SlyshenkovVS, Rakowska M, MoiseenokAG,Wojtczak L. Pantothenic acid and its derivatives protect Ehrlich ascites tumor cells against lipid peroxidation. Free Radic Biol Med. 1995 Dec;19(6):767-72.

12. Ermis H, Parlakpinar H, Gulbas G, Vardi N, Polat A, Cetin A, et al. Protective effect of dexpanthenol on bleomycin-induced pulmonary fibrosis in rats. Naunyn Schmiedebergs Arch Pharmacol. 2013 Dec; 386(12):1103-10.

13. Mihara M, Uchiyama M. Determination of malonaldehyde precursor in tissues by thiobarbituric acid test. Anal Biochem. 1978 May; 86(1):271-8.

14. Sun Y, Oberley LW, LiY.A simple method for clinical assay of superoxide dismutase. Clin Chem. 1988 Mar;34(3):497-500.

15. Aebi H. Catalase. In: Bergmeyer HU, editor. Methods of enzymatic analysis. New York: Academic Press; 1974. p. 673-7.

16. Paglia DE, Valentine WN. Studies on the quantitative and qualitative characterization of erythrocyte glutathione peroxidase. J Lab Clin Med. 1967 Jul;70(1):158-69.

17. Ellman GL. Tissue sulfhydryl groups. Arch Biochem Biophys. 1959 May;82(1):70-7.

18. Erel O. A novel automated direct measurement method for total antioxidant capacity using a new generation, more stable ABTS radical cation. Clin Biochem. 2004 Apr;37(4):277-85.

19. Erel O. A new automated colorimetric method for measuring total oxidant status. Clin Biochem. 2005 Dec;38(12):1103-11.

20. Aycicek A, Erel O, Kocyigit A. Decreased total antioxidant capacity and increased oxidative stress in passive smoker infants and their mothers. Pediatr Int. 2005 Dec;47(6):635-9.

21. Sagit M, Korkmaz F, Akcadag A, Somdas MA. Protective effect of thymoquinone against cisplatin-induced ototoxicity. Eur Arch Otorhinolaryngol. 2013 Aug;270(8):2231-7.

22. Kelly TC, Whitworth CA, Husain K, Rybak LP. Aminoguanidine reduces cisplatin ototoxicity. Hear Res. 2003 Dec;186(1-2):10-6.

23. Huang T, Cheng AG, Stupak H, LiuW, Kim A, Staecker H, et al. Oxidative stress-induced apoptosis of cochlear sensory cells: otoprotective strategies. Int J Dev Neurosci. 2000 Apr-Jun;18(2-3):259-70.

24. Yumusakhuylu AC, Yazici M, Sari M, Binnetoglu A, Kosemihal E, Akdas F, et al. Protective role of resveratrol against cisplatin induced 
ototoxicity in guinea pigs. Int J Pediatr Otorhinolaryngol. 2012 Mar; 76(3):404-8.

25. Ozturan O, Jerger J, Lew H, Lynch GR. Monitoring of cisplatin ototoxicity by distortion-product otoacoustic emissions. Auris Nasus Larynx. 1996;23:147-51.

26. Ichikawa I, Kiyama S, Yoshioka T. Renal antioxidant enzymes: their regulation and function. Kidney Int. 1994 Jan;45(1):1-9.

27. Kizilay A, Kalcioglu MT, Ozerol E, Iraz M, Gulec M, Akyol O, et al. Caffeic acid phenethyl ester ameliorated ototoxicity induced by cisplatin in rats. J Chemother. 2004 Aug;16(4):381-7.
28. Iraz M, Kalcioglu MT, Kizilay A, Karatas E.Aminoguanidine prevents ototoxicity induced by cisplatin in rats. Ann Clin Lab Sci. 2005 Summer;35(3):329-35.

29. Wojtczak L, Slyshenkov VS. Protection by pantothenic acid against apoptosis and cell damage by oxygen free radicals: the role of glutathione. Biofactors. 2003;17(1-4):61-73.

30. Slyshenkov VS, Dymkowska D, Wojtczak L. Pantothenic acid and pantothenol increase biosynthesis of glutathione by boosting cell energetics. FEBS Lett. 2004 Jul;569(1-3):169-72. 